\title{
La flore microbienne et les propriétés fonctionnelles des yaourts et laits fermentés
}

\author{
Aly SAVADOGO* et Alfred S. TRAORE \\ Laboratoire de Microbiologie et de Biotechnologie, Centre de Recherche en Sciences Biologiques, \\ Alimentaires et Nutritionnelles(CRSBAN), Département de Biochimie-Microbiologie (DBM), Unité de \\ Formation et de Recherche en Sciences de la Vie et de la Terre (UFR/SVT), Université de Ouagadougou, \\ Tel/Fax (00226) 50337373 / 03 BP 7131, Ouagadougou, Burkina Faso. \\ *Corresponding author, E-mail: aly.savadogo@univ-ouaga.bf; alysavadogo@gmail.com
}

\section{RESUME}

Cette revue fait le point de l'intérêt dans l'alimentation humaine des yaourts et des laits fermentés par l'étude des caractéristiques de leur flore microbienne et propriétés fonctionnelles. Dans cette revue sont développés les généralités sur les laits fermentés et les différents microorganismes impliqués, les rôles bénéfiques des bactéries lactiques sur la santé et leur résistance aux antibiotiques. La production et la consommation des laits fermentés et des yaourts sont de plus en plus importantes. Cette hausse de la production et de la consommation est liée au développement de l'élevage, à l'éclosion et à la performance des petites et moyennes entreprises laitières. La maitrise de la production des laits fermentés passe par l'utilisation des starters de microorganismes performants qui assureront leurs qualités hygiéniques et nutritionnelles. Les bactéries lactiques sont largement impliquées dans la production des produits laitiers fermentés et les produits fermentés en général du fait de leurs activités métaboliques particulières, qui confèrent aux produits leurs caractéristiques texturales, organoleptiques et de conservation. Dans les pays en développement, la majeure partie du lait issue de l'élevage est transformée par de petites unités et les différentes structures gouvernementales de la santé animale offrent une assistance et des conseils aux divers régions et producteurs.

(C) 2011 International Formulae Group. All rights reserved.

Mots clés : Laits fermentés, Yaourts, Flore microbienne, Propriétés fonctionnelles.

\section{INTRODUCTION}

Les laits fermentés sont largement produits dans de nombreux pays. Ce procédé est l'un des plus vieux utilisés pour augmenter la durée de conservation. Il a été pratiqué par les hommes depuis des milliers d'années. L'origine exacte de la fabrication des laits fermentés est difficile à établir mais il est raisonnable de supposer que cela pourrait dater de plus de 10000 ans, lorsque le mode de vie des hommes a changé, de la collecte à la production des aliments (Tamine, 2002; Tamine, 2008). Ce changement a également inclus la domestication de certains mammifères comme la vache, le mouton, la chèvre, le buffle, le chameau. Il est probable que la transition se soit faite à différentes dates selon les pays. Toutefois, des preuves archéologiques de certaines civilisations comme les Sumériens, les Babyloniens, les 
Egyptiens antiques et les Indiens suggèrent qu'elles étaient bien avancées dans l'agriculture et dans la production des laits fermentés. Il est probable que l'origine de ces produits soit le Moyen orient et les Balkans, et l'évolution des laits fermentés à travers les âges pourrait être attribuée aux compétences culinaires des habitants de ces régions. De nos jours, les laits fermentés sont produits dans de nombreux pays, mais les étapes de fabrication restent encore un procédé complexe qui combine l'art et les sciences antiques (microbiologie, enzymologie, physiques et ingénieries, chimie et biochimie) (Tamime, 2002). Ainsi, il existe un grand nombre de laits fermentés qui diffèrent par leur matière première, leur flore microbienne, leur technologie, leur texture, et leur durée de conservation. Certains sont voisins mais présentés sous des noms variés, beaucoup d'entre eux contiennent l'une ou les deux bactéries spécifiques du yaourt associées à d'autres microorganismes.

La production du lait devient de plus en plus importante, liée au développement de l'élevage. On assiste aujourd'hui à une éclosion de petites et moyennes entreprises de production et de transformation de lait. Il se pose un problème d'assurance qualité pour toutes ces nouvelles structures qui utilisent des moyens modestes.

Cependant, les recherches antérieures réalisées sur les bactéries lactiques ont montré que celles-ci, si elles sont bien exploitées pourraient contribuer à l'amélioration de la qualité et de la conservation des produits fermentés en général et des produits laitiers en particulier.

Le contrôle de la production des aliments fermentés à travers l'utilisation de starters de microorganismes est un facteur important dans l'amélioration de sa stabilité et de sa qualité nutritionnelle et hygiénique.

Les bactéries lactiques (BL) sont largement impliquées dans la fabrication de produits laitiers fermentés du fait de leurs activités métaboliques particulières. La production d'acide lactique est essentielle à la production des produits laitiers fermentés (PLF) et elle leur confère une saveur typique. La production des acides organiques et d'autres composés antimicrobiens, telles les bactériocines, joue un rôle majeur dans la conservation des produits laitiers fermentés et elle contribue à l'inhibition des germes pathogènes ou des contaminants (Desmazeaud, 1996 ; Cherl-Ho, 1997 ; Oyewole, 1997; Daly et Davis, 1998; Ouwehand, 1998 ; Messens, De Vugst, 2002). La transformation du lactose par les ferments lactiques améliore la digestibilité du produit. Les BL contribuent, par leur métabolisme et leurs activités enzymatiques variées, à la production de composés volatils qui participent au développement de l'arôme, de la saveur et de la texture des produits fermentés (Cherl-Ho, 1997 ; Oyewole, 1997). Certaines espèces de bactéries lactiques sont capables au cours de leur métabolisme de produire des exopolysaccharides (EPS) et de les libérer dans le milieu de culture (Cerning et al., 1994; Topisirovic, 1994 ; Cerning, 1990,1995 ; Grobben et al., 1995; Champagne, 1998 ; Dupont, 1998 ; GamarNourani et al., 1998 ; De Vuyst et Degeest, 1999). Les exopolysaccharides interviennent non seulement dans le maintien des propriétés physico-chimiques du lait (texture, viscosité, arômes etc.) mais ils présentent aussi des effets curatifs dans les traitements de certaines maladies gastro-intestinales (Hove et al., 1994 ; Heyman, 2000 ; Simakachorn et al., 2000 ; Isolauri, 2001 ; Soomro et al., 2002).

Depuis quelques années, des fabricants cherchant de nouveaux débouchés ont repris avec l'aide des scientifiques l'idée émise par Metchnikoff (1910) au début du XX $X^{\mathrm{e}}$ siècle que la consommation des laits fermentés peut avoir un effet favorable sur la santé et constituer une "bactériothérapie lactique". C'est ainsi que sont apparus des produits contenant des bactéries intestinales comme les bifidobactéries en association avec des bactéries lactiques.

L'objectif de cette revue est de Contribuer à la connaissance des yaourts et 
des laits fermentés par l'étude des caractéristiques de leur flore microbienne et propriétés fonctionnelles.

\section{Généralités sur les laits fermentés}

Le lait cru est fragile. Pour être conservé, le lait cru doit être pasteurisé, stérilisé, concentré, déshydraté ou transformé en divers produits plus stables (fromages, laits fermentés, etc.). Les laits fermentés, dont font partie les yaourts, sont issus de la fermentation contrôlée du lait sous l'action d'une ou de plusieurs populations bactériennes spécifiques, permettant ainsi sa stabilisation microbiologique en lui conférant une texture et des propriétés organoleptiques et/ou nutritionnelles particulières.

La fermentation du lait par divers types de ferments selon différents procédés est une technique de conservation utilisée traditionnellement dans les pays nordiques (skyr, lattemjölk, tattenjölk, filia, ymer), méditerranéens (naja, mladost, zimme) et dans l'Europe de l'Est (biokys, tarho, kéfir, koumis). Le kéfir, consommé en Europe de l'Est, en Russie et au Moyen-Orient, et le koumis, consommé en Asie centrale, résultent de l'action combinée de bactéries et de levures, et ont la particularité d'être alcoolisés (1-2,5\%). Le plus connu des laits fermentés est cependant le yaourt. Il est originaire de Turquie, de Mongolie, d'Inde, du MoyenOrient et de certaines régions d'Asie. Les noms turc « yoghourt » et grec « yaourt » se sont progressivement imposés depuis le XVe siècle (madzoon en Arménie, laban en Egypte, rhaib en Arabie, kishkh en Jordanie, dahi en Inde). En Europe occidentale, la consommation de laits fermentés et de yaourts a été tardive, mais s'est rapidement répandue, en France, notamment, où leur consommation s'élève à environ $20 \mathrm{~kg}$ par an et par habitant, en moyenne.

Selon la norme CODEX (CODEX STAN 243-2003), les laits fermentés sont des produits obtenus par fermentation du lait par des microorganismes appropriés qui devraient être vivants, actifs et abondants dans le produit fini à la date minimum de péremption (Codex Alimentarius Commission, 2003). Jusque dans les années 1950, la production et/ou la consommation de yaourt était confinée aux communautés du Moyen Orient, des Balkans, de l'Inde, de l'Europe de l'Est, des groupes ethniques vivants dans différentes parties du monde et à ceux qui percevaient que le produit était bénéfique pour la santé. Cependant, il est possible que l'attitude des consommateurs envers le yaourt ait changé éventuellement pour les raisons suivantes :

- la réfrigération est devenue répandue dans le monde entier, le produit a été largement distribué et donc disponible sur le marché

- l'introduction d'une nouvelle génération de yaourts (par exemple l'addition de sucre et de fruits) a donné au produit une image entièrement nouvelle et il est devenu une collation ou un dessert bon marché

- l'incorporation de bactéries probiotiques dans le produit a renforcé les bénéfices santé des laits fermentés (Tamine, 2002).

Actuellement différents laits fermentés sont fabriqués et consommés à travers le monde et approximativement 400 noms génériques désignent des produits traditionnels ou industrialisés (Kurmann et al., 1992). Dans les années 1980, Kurmann (1984) a tenté la classification des laits fermentés dans un "arbre généalogique" qui était basé principalement sur les exigences de croissance optimale des microorganismes du ferment (microflore mésophile ou thermophile). Néanmoins en prenant en compte les microorganismes qui dominent le produit, en incluant leurs principaux métabolites, Robinson et Tamine en 1990 ont proposé un système de classification des laits fermentés comme suit :

$>$ Fermentations lactiques

- De type mésophile, exemples : cultured buttermilk, filmjolk, tattemjolk et langofil ;

- De type thermophile, exemples : yaourt, bulgarian buttermilk, zabadi, dahi; 
- De type thérapeutique ou probiotique, exemples: lait à l'acidophile, yakult, onka, vifit.

Les produits de ce groupe constituent de loin le plus grand nombre de laits fermentés connus dans le monde entier.

$>$ Fermentations lactiques-levures, exemples: kéfir, koumis, lait à l'acidophile et levures

$>$ Fermentations lactiques-moisissures, exemples : vili

\section{Différents laits fermentés et les microorganismes impliqués}

Très bien implantés dans l'alimentation moderne, les laits fermentés bénéficient d'une image forte et possèdent des qualités nutritionnelles reconnues. Riches en calcium, en vitamine $B$ et en acides aminés indispensables, ils renferment des ferments lactiques de plus en plus nombreux et variés, dont l'effet positif sur la microflore intestinale est maintenant largement reconnu.

Les laits fermentés sont des produits laitiers obtenus par la fermentation du lait, lesquels peuvent avoir été fabriqués à base de produits obtenus à partir de lait avec ou sans modification de composition, par l'action de micro-organismes appropriés et résultant de la réduction $\mathrm{du} \mathrm{pH}$ avec ou sans coagulation (précipitation isoélectrique). Ces levains (micro-organismes) doivent être viables, actifs et abondants dans le produit à la date de durabilité minimale. Si le produit subit un traitement thermique après la fermentation, l'exigence portant sur la viabilité des microorganismes ne s'applique plus (FAO/OMS, Codex Alimentarius, 2003).

\section{Le yaourt}

La dénomination yaourt est réservée au lait fermenté uniquement par Streptococcus thermophilus et Lactobacillus delbrueckii subsp. Bulgaricus et en matière de viabilité, la norme précise que la somme des microorganismes constituant le levain doit être au moins de $10^{7}$ UFC $\mathrm{g}^{-1}$. Les normes juridiques existant ou les règlements provisoires sur le yaourt définissent le produit sur la base de la composition chimique ou teneur en matière grasse (entier, semiécrémé/moyen ou écrémé/faible) (FAO/WHO, 1990 ; Tamime et Robinson, 1999).

Au début du XXe siècle, Metchnikoff, collaborateur de Pasteur, montre que le yaourt traditionnel provient de la fermentation du lait sous l'action de deux ferments, Streptococcus thermophilus et une souche de Lactobacillus, généralement Lactobacillus bulgaricus ou Lactobacillus acidophilus. Le yaourt bénéficie aujourd'hui en France d'une définition officielle, protégée et spécifique au produit préparé par culture thermophile protosymbiotique de lait avec ces souches microbiennes. Les souches doivent être vivantes dans le produit fini, à raison d'au moins 10 millions de bactéries par gramme et l'étiquetage doit mentionner le lait dont provient le yaourt s'il ne s'agit pas de lait de vache. La législation reconnaît les deux appellations « yaourt » et « yoghourt ». Les procédés de préparation permettent de modifier la consistance : le yaourt ferme, le yaourt brassé en cuve après la fermentation et qui devient plus onctueux, et le yaourt à boire qui, battu après avoir été brassé, est pratiquement liquide. Un yaourt pèse environ 125 g. L'apport calorique varie de 42 à 115 kcal/100 g. La composition nutritionnelle moyenne d'un yaourt est de $80-90 \%$ d'eau, de 4 à $18 \mathrm{~g} / 100 \mathrm{~g}$ de glucides, de 2,8 à 4,3 g/100 $\mathrm{g}$ de protéines, de 0 à $3,5 \mathrm{~g} / 100 \mathrm{~g}$ de lipides, de sels minéraux, dont du calcium (150 $\mathrm{mg} / 100 \mathrm{~g}$ ), et de vitamines du groupe B. La composition en acides aminés indispensables confère aux protéines laitières une très bonne valeur nutritionnelle. L'apport en calcium est de 110 à $170 \mathrm{mg}$ pour $100 \mathrm{~g}$. Le taux de lipides varie selon que le lait d'origine est entier (35 g/l), 1/2 écrémé $(17,5 \mathrm{~g} / \mathrm{l})$ ou écrémé (de 0 à $1 \mathrm{~g} / \mathrm{l}$ ). La teneur en glucides est augmentée dans les yaourts sucrés, aromatisés ou aux fruits, l'ensemble des adjonctions ne pouvant dépasser $30 \%$ du poids total du 
produit. Les vitamines du groupe B, présentes dans les yaourts et les laits fermentés en quantités intéressantes, proviennent du lait utilisé, mais aussi de ferments lactiques. Les vitamines $\mathrm{A}$ et $\mathrm{D}$ ne sont présentes que dans les produits issus de lait entier.

\section{Le kéfir}

Le kéfir est un lait fermenté originaire de l'Europe de l'Est et est considéré comme étant le yaourt du 21ème siècle (Gorski, 1994). Les informations sur les propriétés salutaires du kéfir et sa valeur nutritionnelle ont encouragé les consommateurs à l'accepter comme un aliment adéquat et les fabricants à accroitre sa production (Kroger, 1993 ; Marshall, 1993 ; Earnworth, 1999). Le kéfir est fait à l'aide d'une microflore unique appelé grain de kéfir, de couleur blanche ou jaune, gélatineuse et de forme irrégulière (Guzel-Seydim et al., 2000). Ces grains de kéfir ont une composition microbiologique complexe et variable comprenant des espèces de levures, de bactéries lactiques et de moisissures. Le kéfir est produit par l'éventail diversifié des espèces microbiennes présentes, les Lactobacilles représentent la plus grande portion $(65-80 \%)$ de la population microbienne (Wouters et al., 2002); les Lactocoques et les levures constituent le reste des microorganismes présents dans le grain de kéfir. Les différents microorganismes et le polysaccharide Kefiran qui composent le grain de kéfir ont été décrits comme une communauté symbiotique qui confère des propriétés uniques au kéfir (Margulis, 1996). Il a été démontré qu'il y'a des espèces spécifiques qu'on rencontre toujours dans les grains de kéfir, en revanche d'autres microorganismes peuvent être présents ou non selon l'origine des grains ainsi que la méthode de culture et les substrats ajoutés (Pintado et al., 1996). Certaines bactéries lactiques qui ont été isolées du kéfir sont Lactobacillus acidophilus, Lactobacillus brevis, Lactobacillus casei, Lactobacillus fermentum, Lactobacillus helveticus, Lactobacillus kefiri,
Lactobacillus parakefiri, Lactococcus lactis et Leuconostoc mesenteroüdes (Assadi et al., 2000).

\section{Le koumis}

Le koumis est un produit laitier fermenté traditionnel originaire des steppes d'Asie Centrale et produit surtout à partir du lait de jument par fermentation spontanée du lactose en acide lactique et alcool (Kosikowski et Mistry, 1997; Ozer, 2000). Le lait de jument possède d'importantes propriétés nutritionnelles et thérapeutiques qui sont bénéfiques aux personnes âgées, convalescentes et aux nourrissons (Marconi et Panfili, 1998).

La composition du lait de jument est significativement différente de celle du lait de vache mais similaire à celle du lait humain en particulier concernant sa faible teneur en azote, son faible ratio caséine/protéines du petit lait et sa teneur élevée en lactose (Yaygin, 1992 ; Bonomi et al., 1994). En outre plusieurs caractéristiques du lait de jument comme son taux élevé en acide polyinsaturés et sa faible teneur en cholestérol semblent supporter l'intérêt croissant pour l'utilisation du lait de jument pour la consommation humaine (Iametti et al., 2001).

Le lait de jument et les produits issus du lait de jument sont largement utilisés en Eurasie en Russie en Mongolie et bien dans d'autres pays du monde (Marconi et Panfili, 1998) et un intérêt considérable a été noté concernant l'utilisation du lait de jument pour la consommation humaine en Europe de l'Ouest (Csapo et al., 1995). Etant donné que le coût du lait de jument est un facteur important pour son utilisation et sa disponibilité étant en deçà de la demande même dans les pays traditionnels d'origine du koumis, l'utilisation du lait de vache pour la production du koumis est d'un grand intérêt pour les chercheurs (Kucukcetin et yaygin, 1999). Cependant, en raison de la différence de composition entre le lait de jument et le lait de vache, il est nécessaire de modifier le lait 
de vache pour le rendre apte à la production de koumis. Quelques chercheurs ont déjà examiné diverses méthodes pour modifier le lait de vache ; par exemple une diminution de la teneur en matière grasse, addition d'eau, de lactose, retentât d'ultrafiltration de lait de vache, lait et/ou acide ascorbique (Kielwein and Daun, 1978; Klupsch, 1985) mais le succès de ces approches a été limité.

\section{Le buttermilk}

Le buttermilk est un sous produit provenant de la fabrication du beurre qui trouve des applications dans plusieurs produits alimentaires. L'utilisation du buttermilk dans les produits alimentaires est étroitement liée à sa composition particulière en composants émulsifiants tels que les phospholipides qui peuvent agir comme des agents émulsifiants dans les vinaigrettes. Cependant, dans la plupart des applications, le buttermilk est utilisé en raison de sa saveur typique comme dans les produits de boulangerie (O'Connell and Fox, 2000).

Un intérêt croissant est montré vis-àvis de ce produit en raison de sa composition qui est unique (Astaire et al., 2003). En effet lorsque les globules gras du lait sont brisés durant le barattage, la membrane recouvrant le noyau lipidique est récupérée dans le buttermilk avec la plupart des protéines, du lactose et des minéraux contenus dans la phase aqueuse de la crème. La membrane du globule gras du lait est riche en différents protéines et phospholipides qui ont un certain potentiel pour les applications fonctionnelles et nutraceutiques. Par exemple il a été montré que la sphingomyéline pourrait contribuer à la prévention de diverses maladies dont le cancer du côlon (Schmelz et al., 2000) et que la phosphatidylcholine pourrait interférer avec le développement des maladies hépatiques (Niederau et al., 1998). Bien que les mécanismes soient encore mal compris il y’a un besoin croissant de fractions enrichies en ces composants pour une étude plus approfondie de leurs effets sur la santé humaine et également dans les systèmes alimentaires.

\section{LES BACTERIES LACTIQUES \\ Caractéristiques générales}

Les bactéries lactiques sont des cellules vivantes, procaryotes, gram-positives, hétérotrophes et chimio-organotrophes. Elles sont le plus souvent immobiles, jamais sporulées, catalase négative, oxydase négative, anaérobies facultatives, micro aérophiles (Tailliez, 2001). Les bactéries lactiques encore appelées bactéries de l'acide lactique sont caractérisées par leur aptitude à fermenter les glucides en produisant de l'acide lactique (D(-), L(+) ou DL) en utilisant les voies cataboliques d'Embden Meyerhof Parnas (EMP), de Dickens-Horecker et d'Entner Doudoroff. Les bactéries lactiques sont dites homofermentaires lorsque l'acide lactique est le seul produit formé; par contre elles sont hétérofermentaires lorsque d'autres composés comme l'éthanol et le $\mathrm{CO}_{2}$ sont produits en même temps. Les bactéries lactiques sont des microorganismes anaérobies qui tolèrent l'oxygène dans une certaine mesure. L'oxygène affecte leur métabolisme mais aussi leur croissance, leur survie et l'intégrité de leur ADN. Les bactéries lactiques possèdent deux types d'oxydases à $\mathrm{NADH}$, ces enzymes catalysent la réduction de $\mathrm{O}_{2}$ en $\mathrm{H}_{2} \mathrm{O}_{2}$ ou de $\mathrm{O}_{2}$ en $\mathrm{H}_{2} \mathrm{O}$. Les bactéries lactiques regroupent un ensemble d'espèces hétérogènes dont le trait commun est la production d'acide lactique. Elles appartiennent à divers genres comme Bifidobacterium, Enterococcus, Lactobacillus, Lactococcus, Leuconostoc, Pediococcus, Streptococcus, Aerococcus, Alloicoccus et Carnobacterium. Elles interviennent dans l'industrie laitière et dans la fermentation de nombreux autres produits alimentaires : saumurage des légumes, boulangerie, fabrication du vin, saurissage des poissons, des viandes et des salaisons, etc. Elles contribuent à la texture, à la saveur des 
aliments et à la production de composés aromatiques.

\section{Le genre Lactobacillus}

Les lactobacilles sont des bactéries lactiques (LAB) Gram-positives qui peuvent être trouvées dans les plantes, les animaux et les niches alimentaires. Certaines espèces associées au tractus gastro-intestinal (GI) sont considérées comme des probiotiques, qui confèrent des avantages de santé à l'hôte. Le genre Lactobacillus est le plus large parmi les bactéries lactiques avec plus de 145 espèces reconnues (Euze'by, 1997). C'est un genre très diversifié, avec une taxonomie qui est généralement considérée comme peu satisfaisante (Canchaya et al., 2006; Makarova et. al, 2006 ; Felis et Dellaglio, 2007). Puisque si peu de protéines semblent être unique dans ces groupes phylogénétiquement proches, nous avons décidé d'enquêter sur l'existence de protéines potentiellement spécifiques à l'habitat, qui n'a pu être trouvée entre les groupes, mais était présente dans certaines niches écologiques. Ces habitats possibles sont le tractus gastro-intestinal (traditionnellement associés à L. acidophilus, L. gasseri, L. johnsonii, L. salivarius et $L$. reuteri), les plantes (L. plantarum, L. brevis, $P$. pentosaceus et Leuconostoc mesenteroides), la viande (L. plantarum, $L$. brevis, L. pentosaceus et $L$. sakei) et les produits laitiers (L. helveticus, L. bulgaricus et L. casei).

\section{Le genre Streptococcus}

Les streptocoques sont des cocci à Gram positif non mobiles, appartenant à la famille des Streptococcaceae. Actuellement, 66 espèces et 12 sous-espèces sont reconnues comme membres du genre Streptococcus (http://www.bacterio.cict.fr/s/streptococcus.ht $\mathrm{ml})$.

Streptococcus thermophilus est perçue comme une bactérie alimentaire qui a récemment émergée et qui a évolué à partir d'un ancêtre commensal par la perte et le gain de fonctions (Delorme et al., 2010).
Streptococcus thermophilus est une bactérie lactique d'importance économique majeure provenant des produits laitiers. Cette espèce est généralement reconnue comme sûre pour les produits alimentaires et le statut de présomption d'innocuité reconnue en Europe lui a été accordé. Elle est historiquement largement utilisée pour la fabrication de yaourt et de fromage en association avec d'autres bactéries lactiques tels que Lactobacillus delbrueckii subsp. bulgaricus et Lactococcus lactis (Hols et al., 2005).

\section{Le genre Lactococcus}

Les lactocoques ont une longue histoire d'utilisation dans les fermentations du lait, des petites opérations traditionnelles dans la ferme ou la maison familiale à des processus à l'échelle industrielle. Il en découle un besoin de pratiques de fabrication plus robustes, plus efficaces et plus affinées qui a conduit à une augmentation des recherches fondamentales et appliquées sur les espèces bactériennes impliquées, en l'occurrence Lactococcus lactis. D'énormes progrès ont été accomplis dans le décryptage de la génétique et la biologie moléculaire de ces microorganismes économiquement très important. De nombreux outils et techniques ont été développés pour disséquer génétiquement $L$. lactis, tel que les méthodes des plasmides vecteurs de clonage et de transformation, les vecteurs de différents gènes (inductibles) d'expression et des méthodes pour introduire des mutations dans des régions déterminées du génome de $L$. lactis. Ainsi, cet organisme est devenu le paradigme des bactéries lactiques (LAB) et la deuxième bactérie à Gram positif la mieux étudiée après Bacillus subtilis. L'augmentation de la connaissance de la génétique, de la physiologie et de la biologie moléculaire de $L$. lactis a permis une profonde compréhension des traits qui sont d'une importance capitale pour les processus industriels dans lesquels l'organisme est utilisé. Par exemple la voie, par laquelle il prend place et métabolise le lactose, conduisant à la chute rapide $\mathrm{du} \mathrm{pH}$ au cours de la fermentation du lait, sa capacité à 
dégrader les protéines et les peptides nécessaires au bon développement de la saveur du fromage ou de sa capacité à produire du diacétyle ont tous été largement étudiés. Cela nous a également permis d'en apprendre davantage au sujet des bactériophages de l'organisme et de prévenir les effets dommageables que ces virus peuvent avoir sur les processus industriels.

\section{Le genre Bifidobacterium}

Le genre Bifidobacterium est probablement le genre le plus étudié de la famille des Bifidobacteriaceae. Les bifidobactéries sont des bactéries Grampositives, anaérobies, hétérofermentaires, non mobiles, non-sporulantes en forme de bâtonnet. En raison de leurs capacités métaboliques, les bifidobactéries sont souvent incluses dans la famille des bactéries lactiques (LAB), même si elles sont phylogénétiquement distinctes avec une teneur en $\mathrm{G}+\mathrm{C}$ allant de $42 \%$ à $67 \%$ (Biavati et Mattarelli, 2001). Le genre Bifidobacterium appartient à la famille des Bifidobacteriaceae, qui comprend également les corynébactéries, mycobactéries et Streptomyces. Environ 30 espèces ont été affectés à ce genre y compris les ajouts récents comme Bifidobacterium denticolens, Bifidobacterium inopinatum isolées des caries dentaires de l'homme (Crociani et al., 1996) et Bifidobacterium psychraerophilum, provenant des intestins de porc (Simpson et al., 2004). A l'exception des espèces isolées à partir de caries dentaires de l'homme, des eaux usées ou les insectes, la majorité des espèces de bifidobactéries sont présentes dans le tractus gastro-intestinal (GIT) des mammifères. Lorsqu'elle est cultivée sur des supports de laboratoire, il génère de l'acétate et de l'acide lactique dans différents rapports molaires selon les conditions de substrat et de croissance (Rasic et Kurman, 1983; Palframan et al., 2003). De petites quantités d'acide formique, d'éthanol et d'acide succinique peuvent aussi être produites, tandis que le dioxyde de carbone, les acides butyrique et proprionique ne sont généralement pas formés (Rasic et Kurman, 1983). Les Bifidobactéries peuvent afficher une gamme de formes de cellules distinctes, notamment des baguettes régulières et diverses formes ramifiées. Le morphotype des cellules de bifidobactéries peut varier selon les espèces, les conditions de culture (Biavati et Mattarelli, 2001) ou de la phase de croissance observée pour Bifidobacterium longum NCC2705. Les bifidobactéries ont d'abord été isolées par Tissier en 1899 dans les matières fécales des nourrissons nourris au sein et leur présence dans l'intestin a depuis lors été associée à un microbiote santé. Cela a favorisé de nombreuses études mettant l'accent sur leur rôle possible dans l'écologie gastro-intestinale et sur la santé, de promouvoir les aspects liés à leur présence dans l'intestin. Aujourd'hui, les bifidobactéries sont devenues économiquement importantes, car elles sont ajoutées en grand nombre comme bactéries vivantes dans de nombreuses préparations alimentaires avec diverses actions liées à la santé.

\section{Le genre Enterococcus}

Le genre Enterococcus regroupe plus de 14 espèces, ce sont des cocci à Gram positif, ovoïdes se présentant en paires ou en courtes chaînettes. La composition de leur paroi, leurs caractères morphologiques, enzymatiques, antigéniques, et leur aptitude à se développer dans des milieux hostiles, permettent de les distinguer des autres cocci à Gram positif. Les entérocoques représentent une proportion importante des bactéries autochtones associées à l'appareil gastrointestinal des mammifères et peuvent causer une infection de cette source endogène. Elles se trouvent essentiellement dans l'intestin et passent dans l'environnement, leur présence dans les eaux est le marqueur d'une pollution fécale. L'incidence des infections causées par les entérocoques, ainsi que la difficulté croissante de traiter ces infections en raison de la multirésistance aux antibiotiques, mettent 
ces organismes, notamment Enterococcus faecium et Enterococcus faecalis, parmi les agents pathogènes émergents de l'homme (Morrison et al., 1997; Robredo et al., 2000). Toutefois, les entérocoques sont omniprésents et se trouvent vivants librement dans le sol, sur les plantes et en grand nombre dans les produits laitiers où dans certains cas, elles prédominent à l'égard des lactobacilles et des lactocoques (Franz et al., 1999 ; Giraffa, 2002). La présence d'entérocoques dans les produits laitiers a longtemps été considérée comme un indicateur de mauvaises conditions sanitaires lors de la production et la transformation du lait. En revanche, de nombreux auteurs suggèrent que les entérocoques peuvent avoir un rôle potentiellement souhaitable dans certains fromages, en raison de leurs activités protéolytiques et lipolytiques, dans le développement des saveurs typiques et pour la production d'enterocins avec une activité antiListeria (Giraffa et al., 1997; Ennahar et Deschamps, 2000). La question importante est de savoir si les entérocoques provenant des aliments et provenant de sources communautaires possèdent un potentiel tout aussi pathogène, ou si les différences dans la pathogénicité existent, peut-être en relation avec les différentes espèces. Dans ce contexte, l'identification correcte des espèces est d'une grande importance tant pour les microbiologistes médicaux qu'alimentaires.

\section{Le genre Leuconostoc}

Les bactéries du genre Leuconostoc sont des bactéries lactiques mésophiles et hétérofermentaires. Ce sont des cocci ovoïdes ou sphériques Gram positif, catalase négative, anaérobies facultatifs et produisant une capsule dans les milieux riches en sucre. Ce genre partage avec le genre Lactobacillus plusieurs points communs et on le trouve dans les mêmes types d'aliments. Plusieurs de ses caractéristiques sont très importantes en alimentation: production d'une saveur agréable, tolérance au sel, habileté à démarrer rapidement la fermentation des produits végétaux (ce qui permet d'inhiber la croissance des bactéries indésirables). La présence de Leuconostoc stimule également la croissance des Lactococcus. Ces deux raisons expliquent pourquoi on inclut les Leuconostoc dans les démarreurs de fermentation pour la production de beurre ou de fromages.

Une espèce, Leuconostoc anos, est importante en vinification (fermentation malolactique). Par contre, les Leuconostoc peuvent également détériorer certains produits alimentaires, en particulier ceux qui sont riches en sucres, avec production de grandes quantités de substances visqueuses. Le genre Leuconostoc s'est enrichi récemment d'un groupe d'espèces appartenant antérieurement au genre Lactobacillus.

\section{LES ROLES BENEFIQUES DES BACTERIES LACTIQUES SUR LA SANTE HUMAINE}

C'est vraisemblablement Metchnikoff qui, le premier vers 1908, a suggéré d'utiliser les laits fermentés contenant une souche de lactobacilles, capables de vivre dans le tractus intestinal, comme composants d'une alimentation utile à la santé humaine.

Les effets bénéfiques des bactéries lactiques pour la santé des consommateurs sont reconnus depuis longtemps. Le zoologiste microbiologiste Ukrainien Ilia llitch (1845-1916) a mis en rapport la longévité de certains peuples, dont les Bulgares et la protection de l'organisme contre plusieurs maladies par consommation de laits fermentés et ainsi l'ingestion de grandes quantités de bactéries lactiques. Pour que les bactéries lactiques puissent avoir un rôle bénéfique sur la santé humaine, il faut qu'elles gardent une certaine activité, voire une viabilité lors du transit intestinal. Ainsi, les bactéries elles-mêmes ou les enzymes doivent pouvoir passer sans dommage irréversible la barrière acide de l'estomac, puis l'effet inhibiteur éventuel des sels biliaires. 
Aussi, de nombreux auteurs (Wood, 1992 ; Meydani et Ha, 2000 ; Marteau et al., 2001 ; Soomro et al., 2002 ; Solis et al., 2002) se sont intéressés, d'une part, à l'influence d'une alimentation à base de produits riches en cultures de microorganismes sur l'écologie du tube digestif et, d'autre part à l'influence sur la santé d'une alimentation avec des produits laitiers contenant des cultures de microorganismes. Actuellement ce sont le yaourt et ses ferments vivants (Streptococcus thermophilus et Lactobacillus delbrueckii subsp. bulgaricus) et les laits fermentés contenant des bifidobactéries (Tamime et al., 1995) et Lactobacillus acidophilus ou $L b$. casei qui ont fait l'objet des recherches les plus approfondies. Les bactéries lactiques peuvent prévenir les maladies gastrointestinales, les diarrhées (Marteau et al., 1998). Guandalini et ses collaborateurs en 2000 ont obtenu une guérison rapide des diarrhées à rotavirus de 287 enfants âgés de 1 à 36 mois administrés avec Lactobacillus rhamnosus GG comparés à ceux qui avaient reçu un placebo.

\section{Effets sur le transit et sur la flore intestinale}

Souvent, les laits acidifiés ou le yaourt sont utilisés pour lutter contre les diarrhées, notamment chez les jeunes enfants, en particulier ceux qui seraient, de plus, mal nourris. L'ingestion de ferments lactiques peut contrer les effets d'une prolifération de certaines souches pathogènes d'Escherichia coli par divers mécanismes: (a) production de substances $\left(\mathrm{H}_{2} \mathrm{O}_{2}\right.$, acides lactique et acétique) directement inhibitrices de E. coli; (b) abaissement du $\mathrm{pH}$ par les acides produits; (c) détoxification par dégradation des entérotoxines; (d) prévention de la synthèse d'amines toxiques; (e) fixation sur le tube digestif empêchant la colonisation de pathogènes, ou effet barrière par compétition métabolique s'il n'y a pas d'attachement. Lactobacillus bulgaricus ne s'implante pas dans le tube digestif et y survit difficilement à cause de sa faible tolérance aux sels biliaires, de son peu de résistance aux $\mathrm{pH}$ acides. En revanche, Streptococcus thermophilus, et surtout Lb. acidophilus, survivent beaucoup mieux dans l'intestin. Ce dernier germe a fait l'objet de développements industriels récents car il a été montré qu'il pouvait s'opposer à la prolifération de Staphylococcus aureus, de Salmonella typhimurium, de E. coli entéropathogène ou de Clostridium perfringens. Plusieurs mécanismes peuvent être envisagés: d'une part, certaines souches de $L b$. acidophilus produisent du peroxyde d'hydrogène et, d'autre part, elles peuvent sécréter des substances peptidiques de type bactériocines dénommées «lactacin $B »$, «lactocidin» ou «lactacin $F$ ». Un autre aspect intéressant est l'effet des bactéries lactiques sur le métabolisme de la flore intestinale normale. Elles peuvent abaisser les quantités de certaines enzymes ( $\beta$-glucuronidase, azoréductase ou nitroréductase) formées par cette flore. La réduction des activités de ces trois enzymes de la flore intestinale est intéressante, car elles sont associées à la formation de carcinogènes. Des souches de Bifidobacterium bifidum possèdent aussi des capacités d'inhibition de flore pathogène comme Clostridium difficile.

\section{Amélioration de l'intolérance au lactose et de la digestion du lactose}

L'apparition de symptômes digestifs après ingestion de lait peut être liée au lactose notamment par l'incapacité de le digérer par manque de lactase de la muqueuse intestinale. Chez les adultes, les symptômes digestifs d'intolérance au lactose sont principalement des douleurs abdominales, crampes, flatulences. Chez le jeune enfant, l'importance clinique de l'intolérance au lactose est plus grande, avec diarrhées acides et selles contenant des sucres réducteurs. Il a été clairement démontré que le yaourt permet l'absorption du lactose chez les sujets déficients en lactase et qu'il améliore les symptômes digestifs d'intolérance au lactose. 
Il faut noter que ces effets bénéfiques disparaissent lorsque le yaourt a subi un traitement thermique. Ceci signifie que l'action favorable n'existe que si les bactéries sont vivantes et leur lactase ( $\beta$-galactosidase) actives. Un chauffage rapide du yaourt inhibe les bactéries lactiques et la lactase. Le genre Bifidobacterium a un type fermentaire acétique-lactique-formique intéressant du fait que l'acide lactique formé est de forme $\mathrm{L}(+)$. Or, on sait que cette forme $\mathrm{L}(+)$ est totalement métabolisée et qu'il ne faut pas craindre, chez les jeunes enfants, certains inconvénients qui peuvent être observés avec les formes DL ou $\mathrm{D}(-)$ produites par les lactobacilles.

La production d'acide lactique, la lipolyse et la protéolyse effectuées par les bactéries lactiques stimuleraient la digestion des aliments. L'intolérance au lactose observée chez certaines personnes peut être combattue par l'utilisation des laits fermentés comme le yaourt dans lequel le lactose est dégradé en acide lactique mais aussi à cause de l'apport en $\beta$-galactosidase. La flore intestinale endogène exerce de nombreuses fonctions physiologiques, telles que des métabolismes (fermentation...) et un effet de barrière s'opposant à la colonisation par des microorganismes pathogènes. La résultante pour l'hôte est souvent bénéfique mais parfois néfaste.

\section{Activité hypocholestérolémiante}

Trois observations principales expliquent l'activité hypocholestérolémiante des probiotiques :

(a) La première observation date de 1974, lorsque Mann et Spoerry (1974) démontrèrent que chez les tribus Masaï, qui pratiquent l'élevage du bétail, le taux bas de cholestérol dans le sang s'explique par la consommation journalière de lait fermenté par des souches sauvages de bactéries lactiques.

(b) La deuxième observation est celle de Eyssen (1973) qui a montré par des expériences menées sur des animaux «axéniques » que la microflore intestinale a un effet direct sur la teneur en cholestérol du sang.

(c) La troisième observation est de Gilliland (1985) qui a montré que certaines souches de bactéries lactiques ont la capacité d'assimiler le cholestérol.

Les probiotiques semblent également posséder une action anticholestérolémiante. En effet, certaines bactéries lactiques inhiberaient la conversion de l'acétate en cholestérol. L'ingestion de Lb acidophilus diminue le taux de cholestérol dans le sérum sanguin de la rate.

\section{Action anticarcinogène et action sur le système immunitaire}

Les bactéries lactiques semblent provoquer des réactions immunitaires in vivo. Une réaction spécifique est obtenue avec une espèce de $L b$ casei qui provoque une stimulation contre une infection à Salmonella typhimurium. Le yaourt peut servir de produit préventif contre les diarrhées à Salmonella et à E. coli. Les probiotiques stimuleraient la production d'immunoglobuline (Production d'Ig G2 chez des souris suite à l'ingestion de yaourt). Le yaourt aurait un effet inhibiteur sur la prolifération des cellules cancéreuses en culture.

Plusieurs études ont démontré que les EPS ont une activité anti-cancérigène. Kitazawa et ses collaborateurs (1991) ont postulé que l'EPS produit par Lactococcus lactis ssp. cremoris KVS 20 est responsable d'un effet anti-tumoral de cette souche. Ceci a été observé lors d'injections intrapéritonéales chez des souris. Dans une étude subséquente, Kitazawa et ses collabrateurs (1996) ont attribué à un exophosphopolysaccharide produit par cette souche une activation des macrophages et l'induction de la production de cytokine.

Les mêmes auteurs ont fractionné l'EPS (Exopolysaccharide) produit par $L b$. delbrueckii ssp. bulgaricus OLL 1073R-1 en un polysaccharide neutre et un polysaccharide acide. La fraction acide composée de glucose, 
galactose et phosphore a démontré une activité mitogénique sur les lymphocytes B, dirigée par le groupement phosphate. Une activité mitogénique a aussi été observée in vitro pour un polysaccharide d'une taille de $1,5 \times 10^{6} \mathrm{Da}$ produit par Bifidobacterium adolescentis M101-4 (Hosono et al., 1997). Un effet anti-cancérigène a été trouvé in vitro et in vivo pour les polysaccharides capsulaires de Bifidobacterium breve YIT4014 et 4043 et Bifidobacterium bifidum YIT 4007 (Nagaoka et al., 1994). Ces polysaccharides étaient composés principalement de rhamnose. Il a été montré que les polysaccharides contenant plus de $60 \%$ de rhamnose se sont montrés plus efficaces pour le traitement du cancer gastrointestinal. Tous les travaux mentionnés jusqu'ici ont été fait in vitro ou par injection de l'EPS(Exopolysaccharide) à des rats ou des souris. Très peu d'expériences ont été réalisées in vivo avec administration orale de l'EPS. Zubillaga et al. (2001) ont administré l'EPS(Exopolysaccharide)KGF-C oralement. Les auteurs ont observé un retardement de la croissance des cellules cancéreuses par cet EPS soluble dans l'eau et extrait de grains de kéfir.

\section{Les probiotiques et prebiotiques}

Les probiotiques sont des microorganismes ingérés vivants capables d'exercer des effets bénéfiques sur leur hôte. Il s'agit le plus souvent de bactéries ou de levures présentes soit dans les aliments notamment les produits laitiers fermentés, soit dans des médicaments et volontiers sous forme lyophilisées (Marteau, 1998). Les genres bactériens les plus utilisés sont Bifidobacterium, Lactobacillus, Enterococcus et Saccharomyces.

Les prébiotiques sont des ingredients alimentaires non digestibles qui ont des effets bénéfiques sur leur hôte en stimulant de façon selective la croissance et/ou l'activité d'une ou plusieurs bactéries présentes dans le côlon. Les prébiotiques favorisent le développement des probiotiques dans l'intestin. Les probiotiques peuvent être considérés comme un moyen de véhiculer des principes actifs qu'ils contiennent (enzymes, composants de paroi, peptides immunomodulateurs, substances antibactériennes...) jusqu'à leurs cibles d'action dans le tractus digestif. Les critères de sélection des bactéries pour la constitution des probiotiques sont: non cariogénique, non pathogène, résistante aux enzymes digestives buccales et gastriques, au pH acide de l'estomac aux sels biliaires, avoir une capacité d'adhérence aux cellules intestinales pour coloniser le tractus intestinal sans perturber la flore intestinale normale. La consommation du lait à Lactobacillus acidophilus faciliterait le transit intestinal et combattrait la constipation. L'équilibre de la flore intestinale peut être rétabli par une consommation régulière du lait ou de yaourt à $L b$ acidophilus suite aux troubles causés par des antibiotiques. Les $L b$ acidophilus auraient cette aptitude à coloniser l'intestin et diminuent le nombre de Clostridies.

\section{BACTERIES LACTIQUES ET RESISTANCE AUX ANTIBIOTIQUES}

La découverte et le développement d'antibiotiques classiques, qui étaient principalement à base de composés antimicrobiens générés par des bactéries ou des champignons, ont conduit à des améliorations spectaculaires dans la capacité à traiter les maladies infectieuses et une augmentation significative de la production d'aliments d'origine animale. Sans aucun doute, les antibiotiques représentent une des principales avancées scientifiques et médicales du $20^{\text {ème }}$ siècle (Gordon et al., 2005; McPhee et Hancock, 2005).

$\mathrm{Au}$ cours des 50 dernières années, l'espérance de vie humaine et la qualité de vie ont considérablement augmenté en raison de l'amélioration de la nutrition et l'utilisation d'antibiotiques dans la lutte contre les maladies infectieuses. Toutefois, l'âge d'or du traitement aux antibiotiques est en déclin en 
raison de l'apparition et la propagation de la résistance chez les microorganismes nocifs.

À l'heure actuelle, il ya une grande préoccupation que les populations bactériennes commensales de la nourriture et $\mathrm{du}$ tractus gastro-intestinal de l'homme et des animaux, telles que les bactéries lactiques et les bifidobactéries, pourraient agir comme un réservoir de gènes de résistance aux antibiotiques. Cette résistance pourrait finalement être transférée aux bactéries pathogènes et opportunistes de l'homme entravant ainsi le traitement des infections. Les espèces de bactéries lactiques ont été traditionnellement utilisées comme ferments dans la production d'aliments pour animaux et des denrées alimentaires. De plus, les bactéries lactiques et les bifidobactéries sont des habitants normaux du tractus gastrointestinal, où ils sont reconnus exercer des effets favorables à la santé, et des souches sélectionnées sont actuellement utilisées comme probiotiques. Les gènes de résistance aux antibiotiques portés par les bactéries lactiques et les bifidobactéries peuvent être transférés à des bactéries pathogènes de l'homme soit pendant la fabrication des aliments ou lors du passage dans le tube digestif.

\section{PROPRIETES ANTIMICROBIENNES DES BACTERIES LACTIQUES}

Bien que le traitement aux antibiotiques soit toujours le premier choix pour lutter contre les infections microbiennes chez les humains et les animaux, la prévalence de la résistance bactérienne aux antibiotiques classique est une préoccupation croissante de santé publique. Ceci a conduit à la recherche de nouveaux antimicrobiens qui sont généralement efficaces et moins susceptibles d'induire une résistance aux antimicrobiens. Les peptides antimicrobiens naturels, codés par les gènes sont un groupe diversifié de molécules immunitaires innées présentes dans tous les organismes. Les peptides antimicrobiens (PAM) d'âge mûr contiennent généralement 7 à 100 résidus d'acides aminés, possèdent une charge nette positive et une structure amphiphile qui facilite l'interaction avec les membranes microbiennes négativement chargées ou autres cibles cellulaires (Yeaman et Yount, 2007; Linde et al., 2008; Sang et Blecha, 2008). Par rapport aux antibiotiques classiques, qui sont généralement actifs contre les bactéries ou les champignons, les PAM exercent souvent une activité contre un large spectre de microorganismes comme les bactéries, champignons, parasites, virus enveloppés et même certaines cellules cancéreuses. En outre, contrairement aux antibiotiques classiques, qui ont généralement pour cible une enzyme du métabolisme et peuvent sélectivement induire une résistance à des micro-organismes, les peptides antimicrobiens tuent les microbes principalement par des mécanismes de ciblage à la membrane formant des pores, un mécanisme qui est intrinsèquement plus difficile aux microbes de contourner par le développement de la résistance (Boman, 2003; Hancock et Sahl, 2006). Les peptides antimicrobiens ont été isolé de la plupart des formes de vie et comprennent les bactériocines, peptides antibiotiques fongiques, thionines et défensines des végetaux, les défensines et cécropines d'insectes, temporins et magainines des amphibiens ainsi que les défensines et cathélicidines des vertébrés supérieurs (McPhee et Hancock, 2005; Yeaman et Yount, 2007).

Certaines espèces de bactéries lactiques comme les $L b$ brevis, Lb plantarum, Lb acidophilus, Lb delbrueckii subsp. bulgaricus produisent des antibiotiques actifs contre les bactéries Gram positives (Bacillus, Staphylococcus, Streptococcus, Sarcina) ou Gram négatives (Pseudomonas, Escherichia, Shigella, Salmonella, Serretia, Proteus, Vibrio).

L'élimination de bactéries pathogènes intervient suite à la production de bactériocines, à une acidification du milieu 
qui entraîne une inhibition du métabolisme oxydatif et une diminution de $\mathrm{pH}$ intracellulaire.

Les bactériocines sont produites par les bactéries, ce sont de petits peptides, thermostables que les bactéries utilisent dans le cadre de la compétition contre d'autres bactéries de la même espèce (spectre étroit) ou contre des bactéries d'autres genres (large spectre) (Cotter et al., 2005). Une ou plusieurs bactériocines ont été identifiées, ou on croit qu'elles existent dans toutes les espèces de bactéries et d'archaebactéries (Cotter et al., 2005; Willey et van der Donk, 2007).

Les bactériocines les plus prometteuses dans le développement d'antibiotiques sont celles produites par les bactéries lactiques (LAB), avec les genres de base, notamment Lactobacillus, Lactococcus, Leuconostoc, Pediococcus et Streptococcus. En raison de la longue histoire d'utilisation des bactéries lactiques dans la transformation des aliments fermentés et les informations de sécurité, les effets antimicrobiens des bactéries lactiques pour la conservation des aliments sont largement acceptés. De plus, les bactéries lactiques sont largement utilisées comme probiotiques dans les aliments pour la transformation et la conservation (De Vuyst et Leroy, 2007; Sit et Vederas, 2008). Les bactériocines dérivées des bactéries lactiques entreront probablement dans la pharmacopée par voie orale ou comme antibiotiques gastrointestinaux (Rossi et al., 2008). Exemples de bactériocines dérivées des bactéries comprennent la nisine, mersacidine, lacticine 481 et lacticine 3147. Parmi ces derniers, la nisine a été approuvé pour une utilisation commerciale dans le traitement de certains aliments et comme anti-infectieux pour les mammites bovines (Cotter et al., 2005; Dufour et al., 2007) et la mersacidine a été évaluée dans des essais précliniques pour le traitement d'infections causées par des bactéries à Gram-positif (Hancock et Sahl, 2006). La Lacticine 3147, un antibiotique à deux peptides, s'est montré prometteur pour prévenir les mammites (Crispie et al., 2005) et comme conservateur alimentaire (Gardiner et al., 2007). Il est important de noter que plusieurs bactériocines notamment la lacticine 3147, la mersacidine et la leucocine A ont montré une activité efficace contre des souches bactériennes résistantes aux antibiotiques telles que les entérocoques vancomycine résistants et les Staphylococcus aureus résistants à la méthicilline (Kruszewska et al., 2004 ; Sit et Vederas, 2008). En plus des bactéries lactiques d'origine alimentaires, les bactéries lactiques commensales du tube gastro-intestinal (entérocoques et streptocoques) et la microflore de la famille des Enterobacteriaceae produisent un large panel de bactériocines, telles que les enterocins, salivaricins, colicines et microcins. Ces bactériocines ont un potentiel probiotique significatif ou peuvent être utilisées comme antibiotiques après des modifications biotechnologiques appropriées (Duquesne et al., 2007; Nes et al., 2007). Song et Richard en 1997 ont montré chez Listeria, bactéries à Gram positif, que les cellules résistantes aux bactériocines ont une membrane de composition différente de celle des cellules sensibles.

\section{Conclusion}

Les bactéries lactiques sont parmi les bactéries à Gram-positif les plus étudiées sur le plan génétique et moléculaire après Bacillus subtilis en particulier à cause de leur importance dans l'industrie agro-alimentaire. Les bactéries lactiques sont dépendantes des sucres comme source d'énergie ; cette source de carbone est transformée principalement en lactate. Les bactéries lactiques, en raison de leurs caractères technologiques, nutritionnels et éventuellement thérapeutiques, jouent un rôle central dans le traitement du lait pour obtenir des produits fermentés comme les fromages et les yaourts. Par la fermentation, les bactéries lactiques confèrent les propriétés 
organoleptiques et rhéologiques particulières aux produits laitiers fermentés.

\section{REMERCIEMENTS}

Nous exprimons notre gratitude à SEMDE Zenabou pour son soutien technique lors de l'élaboration de ce travail ; elle a bien voulu contribuer à la saisie du texte et à la recherche des informations.

\section{BIBLIOGRAPHIE}

Assadi MM, Pourahmad R, Moazami N. 2000. Use of isolated kefir starter cultures in kefir production. World $J$. Microbiol. Biotech., 16: 541-543.

Astaire JC, Ward R, German JB, JimenezFlores R. 2003. Concentration of polar MFGM lipids from buttermilk by microfiltration and supercritical fluid extraction. J. Dairy Sc., 86: 2297-2307.

Biavati B, Mattarelli P. 2001. The family Bifidobacteriaceae. In The Prokaryotes, Dworkin M, Falkow S, Rosenberg E, Schleifer KH, Stackebrandt E (eds). Springer: New York; 1-70.

Boman HG. 2003. Antibacterial peptides: basic facts and emerging concepts. J. Int. Med., 254: 197-215.

Bonomi F, Iametti S, Pagliarini E, Solaroli G. 1994. Thermal sensitivity of mares' milk proteins. J. Dairy Research., 61: 419422.

Canchaya C, Claesson MJ, Fitzgerald GF, Van Sinderen D, O'Toole PW. 2006. Diversity of the genus Lactobacillus revealed by comparative genomics of five species. Microbiol., 152: 31853196.

Cerning J, 1990. Exocellular polysaccharides produced by lactic acid bacteria. FEMS Microbiol. Rev., 87:113-130.

Cerning J, Renard CMGC, Thibault J F, Bouillanne C, Landon M, Desmazeaud M, Topisirovic L. 1994. Carbon source requirements for exopolysaccharide production by Lactobacillus casei CG11 and partial structure analysis of the polymer. Appl. Env. Microbiol., 60: 3914-3919.

Cerning J. 1995. Production of exopolysaccharides by lactic acid bacteria and dairy propionibacteria. Lait, 75: 463-472.

Champagne PC. 1998. Production de Ferments Lactiques dans l'Industrie Laitière. EDISEM : Canada ; 210.

Cherl-Ho L. 1997. Lactic acid fermented foods and their benefits in Asia. Food Control., 8: 259-269.

Crociani F, Biavati B, Alessandrini A, Chiarini C, Scardovi V. 1996. Bifidobacterium inopinatum sp. nov. and Bifidobacterium denticolens sp. nov., two new species isolated from human dental caries. Int. J. Syst. Bacteriol. 46: 564-571.

Csapos J, Stefler J, Martin T G, Makray S, Csapo-Kiss Z. 1995. Composition of mares' milk colostrum and milk. Fat content, fatty acid composition and vitamin content. Int. Dairy J., 5: 393402.

Cotter PD, Hill C, Ross RP. 2005. Bacteriocins: developing innate immunity for food. Nat. Rev. Microbiol., 3: 777-788.

Crispie F, Twomey D, Flynn J, Hill C, Ross P, Meaney W. 2005. The lantibiotic lacticin 3147 produced in a milkbased medium improves the efficacy of a bismuth-based teat seal in cattle deliberately infected with Staphylococcus aureus. J. Dairy Research, 72: 159-167.

Daly C, Davis R. 1998. The biotechnology of lactic acid bacteria with emphasis on applications in food safety and human health. Agri. food Sci. Finland, 7: 251264.

Delorme C, Bartholini C, Bolotine A, Ehrlich SD, Renault P. 2010. Emergence of a cell wall protease in the Streptococcus thermophilus population. Apl. Env. Microbiol., 76(2): 451-460. 
Desmazeaud M. 1996. Les bactéries lactiques dans l'alimentation humaine : utilisation et innocuité. Cahiers Agric., 5: 331-343.

De Vuyst L, Degeest B. 1999. Heteropolysaccharides from lactic acid bacteria. FEMS Microbiol. Rev., 23: 153177.

De Vuyst L, Leroy F. 2007. Bacteriocins from lactic acid bacteria: production, purification, and food applications. $J$. Molec. Microbiol. Biotech., 13: 194199.

Dufour A, Hindre' T, Haras D, Le Pennec JP. 2007. The biology of lantibiotics from the lacticin 481 group is coming of age. FEMS Microbiol. Rev., 31: 134-167.

Dupont I. 1998. Identification moléculaire de souches de lactobacilles productrices d'exopolysaccharides et comparaison de la production d'exopolysaccharides par trois de ces souches. Thèse, Université Laval, Canada.

Duquesne S, Destoumieux-Garzo'n D, Peduzzi J, Rebuffat S. 2007. Microcins, gene-encoded antibacterial peptides from enterobacteria. Nat. Prod. Rep., 24: 708734.

FAO/WHO. 1990. Codex AlimentariusAbridged Version, Joint $\mathrm{FAO}=\mathrm{WHO}$ Food Standards Program-Codex Alimentarius Commission, ed. BL Smith. Rome: Food and Agricultural Organization of the United Nations.

FAO /OMS. 2003. Codex Alimentarius, Code d'usages international recommandé principes généraux d'hygiène alimentaire, Cac/rcp 1-1969, rév. 4.

Earnworth ER. 1999. Kefir: from folklore to regulatory approval. J. Nutrac. Funct. Med. Foods. 1: 57-68.

Felis GE, Dellaglio F. 2007. Taxonomy of lactobacilli and bifidobacteria. Curr Issues Intest Microbiol., 8: 44-61.

Gamar-Nourani L, Blondeau K, Simonet J M. 1998. Influence of culture conditions on exopolysaccharide production by
Lactobacillus rhamnosus strain C83. J. Appl. Microbiol., 85: 664-672.

Gardiner GE, Rea MC, O'Riordan B, O'Connor P, Morgan SM, Lawlor PG, Lynch PB, Cronin M, Ross RP, Hill C. 2007. Fate of the two-component lantibiotic lacticin 3147 in the gastrointestinal tract. Appl. Env. Microbiol., 73: 7103-7109.

Gordon YJ, Romanowski EG, McDermott AM. 2005. A review of antimicrobial peptides and their therapeutic potential as anti-infective drugs. Current Eye Research, 30: 505-515.

Gorski D. 1994. Kefir: 21st century yogurt? Dairy Foods, 95(2) : 49.

Grobben GJ, Sikkema J, Smith MR, De Bond JAM. 1995. Production of extracellular polysaccharides by Lactobacillus delbrueckii subsp. bulgaricus NCFB 2772 grown in a chemically defined medium. J. Appl. Bacteriol., 79: 103107.

Guzel-Seydim Z, Seydim AC, Greene AK. 2000. Organic acids and volatile flavor components evolved during refrigerated storage of kefir, J. Dairy Sc., 83: 275277.

Hancock RE, Sahl HG. 2006. Antimicrobial and host-defense peptides as new antiinfective therapeutic strategies. Nat. Biotech., 24: 1551-1557.

Heyman M. 2000. Effect of lactic acid bacteria on diarrheal diseases. Am. J. Clin. Nutr., 19 :137-146.

Hols P, Hancy F, Fontaine L, Grossiord B, Prozzi D,. Leblond-Bourget N, Decaris B, Bolotin, Delorme C, Dusko Ehrlich S, Gue'don E, Monnet V, Renault P, Kleerebezem M. 2005. New insights in the molecular biology and physiology of Streptococcus Thermophilus revealed by comparative genomics. FEMS Microbiol. Rev., 29: 435-463.

Hove H, Nordgaard-Andersen I, Brobech Mortensen P. 1994. Effect of lactic acid bacteria on the intestinal production of 
lactate and short-chain fatty acids, and the absorption of lactose. Am. J. Clin. Nutr., 59: 74-79.

Isolauri E. 2001. Probiotics in human disease. Am. J. Clin. Nutr., 73: 1142-1146.

Ennahar S, Deschamps S. 2000. Anti-Listeria effect of enterocin A, produced by cheese-isolated Enterococcus faecium EFM01, relative to other bacteriocins from lactic acid bacteria. J. Appl. Microbiol., 88: 449-457.

Franz CMAP, Holzapfel WH, Stiles ME. 1999. Enterococci at the crossroads of food safety? Int. J. Food Microbiol., 47: $1-24$.

Giraffa G. 2002. Enterococci from foods. FEMS Microbiol Rev., 26: 163-171.

Giraffa G, Carminati D, Neviani E. 1997. Enterococci isolated from dairy products: a review of risks and potential technological use. J. Food Prot., 60: 732-738.

Iametti S, Tedeschi G, Oungre E, Bonomi F. 2001. Primary structure of k-casein isolated from mares' milk. J. Dairy Research., 68: 53-61.

Kielwein G, Daun U. 1978. Ein neues Getraenk nach Nomadenart auf der Basis von Kuhmilcheiwei. Deutsche Molkerei Zeitung, 99: 724-726.

Klupsch HJ. 1985. M. oglichkeiten zur industriellen Herstellung von Kumys aus Kuhmilch. Deutsche Molkerei Zeitung, 11: 293-296.

Kroger MK. 1993. Kefir. Cult. Dairy Prod. J. 26: $28-29$.

Kruszewska D, Sahl HG, Bierbaum G, Pag U, Hynes SO Ljungh A. 2004. Mersacidin eradicates methicillin - resistant Staphylococcus aureus (MRSA) in a mouse rhinitis model. J. Ant. Chemotherap., 54: 648-653.

Kosikowski FV, Mistry VV. 1997. Cheese and fermented milk foods, 1(3): 10, 27, 65-67. Westport: F.V. Kosikowski, L.L.C.
Kucukcetin A, Yaygin H. 1999. Studies on the properties of the koumiss made from original mares' and whey powder added cows' and goats'. Special Issue for the 11th congress of KU. KEM Biotechnology, Isparta, Turkey, 23(2): 29-35.

Kurmann JA, Rasic JLj, Kroger M. 1992. Encyclopedia of Fermented Fresh Milk Products. Van Nostrand Reinhold: New York.

Kurmann JA. 1984. Aspects of the production of fermented milks. Fermented Milks. Document 179, International Dairy Federation, Brussels, Belgium ; 16-26.

Linde A, Ross CR, Davis EG, Dib L, Blecha F and Melgarejo T. 2008. Innate immunity and host defense peptides in veterinary medicine. J. Veter. Internal Med., 22: 247-265.

Makarova K, Slesarev A, Wolf Y, Sorokin A, Mirkin B, Koonin E, Pavlov A, Pavlova N, Karamychev V, other authors. 2006. Comparative genomics of the lactic acid bacteria. Proc Natl Acad Sci USA, 103: 15611-15616.

Marconi E, Panfili G. 1998. Chemical composition and nutritional properties of commercial products of mare milk powder. J. Food Comp. Anal., 11: 178187.

Margulis L. 1996. From kefir to death. In How Things Are, Brockman J, Matson K (Eds). William Morrow and Co: New York; 69-78.

Marshall VM. 1993. Starter cultures for milk fermentation and their characteristics. $J$. Soc. Dairy Technol. 46: 49-56.

Metchnikoff E. 1910. Prolongation of Life (revised edn of 1907, tran.), Mitchell C (ed.). Heinemann: London.

Messens W, De Vuyst L. 2002. Inhibitory substances produced by lactobacilli isolated from sourdoughs - a review. Int. J. Food Microb. 72: 31-43. 
McPhee JB, Hancock RE. 2005. Function and therapeutic potential of host defence peptides. J. Peptide Sc., 11: 677-687.

Morrison D, Woodford N, Cookson B. 1997. Enterococci as emerging pathogens of humans. Soc. Appl. Bacteriol. Symp Ser., 26: 89S-99S.

Nes IF, Diep DB, Holo H. 2007. Bacteriocin diversity in Streptococcus and Enterococcus. J. Bact., 189: 1189-1198.

Niederau C, Strohmeyer G, Heintges T, Peter K, Gopfert E. 1998. Polyunsaturated phosphatidyl-choline and interferon alpha for treatment of chronic hepatitis $b$ and c: A multi-center, randomized, double-blind, placebo-controlled trial. Leich study group. Hepatogastroenter., 45: 797-804.

O'Connell JE, Fox PF. 2000. Heat stability of buttermilk. J. Dairy Sc., 83: 1728-1732.

Ouwehand AC. 1998. Antimicrobial components from lactic acid bacteria. In Lactic Acid Bacteria: Microbiology and Functional Aspects, (vol. 2). Marcel Dekker Inc.: New York; 139-159.

Oyewole OB. 1997. Lactic fermented food in Africa and their benefits. Food Control., 8: 289297.

Ozer B. 2000. Fermented milks. Products of Eastern Europe and Asia. In Encyclopedia of Food Microbiology, Robinson RK, Batt CA, Patel PD (eds). Academic Press: London; 803-804.

Palframan RJ, Gibson GR, Rastall RA. 2003. Carbohydrate preferences of Bifidobacterium species isolated from the human gut. Curr. Issues Intest. Microbiol., 4: 71-75.

Pintado ME, Lopes Da Silva JA, Fernandes PB, Malcata FX, Hogg TA. 1996. Microbiological and rheological studies on Portuguese Kefir grains, Int. J. Food Sci. Tech., 31: 15-26.

Rasic LJ, Kurman JA. 1983. Bifidobacteria and Their Role. Birkhäuser Verlag: Basel, Boston, Stuttgart.
Robinson RK, Tamine AY. 1990. Microbiology of fermented milks. In The Microbiology of Milk Products Dairy Microbiology (vol. 2), Robinson RK (Ed). Els.: London; 291-343.

Robredo B, Singh K V, Baquero F, Murray B E, Torres C. 2000. Vancomycin-resistant enterococci isolated from animals and food. Int. J. Food Microbiol., 54: 197204.

Rossi LM, Rangasamy P, Zhang J, Qiu XQ and Wu GY 2008. Research advances in the development of peptide antibiotics. $J$. Pharmaceutical Sc., 97: 1060-1070.

Sang Y, Blecha F. 2008. Porcine host defense peptides: expanding repertoire and functions. Developmental and Comparative Immunology, 2008 June 9 [Epub ahead of print] doi:10.1016/j.dci.2008.05.006.

Schmelz EM, Sullards MC, Dillehay DL, Merrill AHJ. 2000. Colonic cell proliferation and aberrant crypt foci formation are inhibited by dairy glycosphingolipids in 1, 2dimethylhydrazinetreated cf1 mice. $J$. Nutr., 130: 522-527.

Simakachorn N, Pichaipat V, Rithipornpaisarn P, Kongkaew C, Tongpradit P, Varavithya W. 2000. Clinical evaluation of the addition of lyophilised, heat-killed Lactobacillus acidophilus $L B$ to oral rehydratation therapy in the treatment of acute diarrhea in children. J. Pediatr. Gastroenterol. Nutr., 30: 68-72.

Simpson PJ, Ross RP, Fitzgerald GF, Stanton C. 2004. Bifidobacterium psychraerophilum sp. nov. and Aeriscardovia aeriphila gen. nov., sp. nov., isolated from a porcine caecum. Int. J. Syst. Evol. Microbiol., 54: 401406.

Sit CS, Vederas JC. 2008. Approaches to the discovery of new antibacterial agents based on bacteriocins. Biochem. Cell Biol., 86: 116-123. 
Soomro AH, Masud T, Anwaar Kiran. 2002. Role of lactic acid bacteria (LAB) in food preservation and Human health. A Review. Pakistan J. Nut., 1(1): 20-24.

Song HJ, Richard J. 1997. Antilisterial activity of three bacteriocins used at sub minimal inhibitory concentrations and cross-resistance of the survivors. - Int. $J$. Food Microbiol., 36(2): 155-161.

Tamime AY. 2008. Milk Processing and Quality Management. Wiley Blackwell.

Tamime AY. 2002. Fermented milks: a historical food with modern applicationsa review. Eu. J. Clinical Nut., 56(4): S2S15.

Tamime AY, Robinson RK. 1999. Yoghurt: Science and Technology (2nd edn). CRC Press, Boca Raton, FL.

Tissier MIH. 1899. Compte rendu. Soc. Biol., 51: $943-94$
Topisirovic L. 1994. Carbon source requirements for exopolysaccharide production by Lactobacillus casei CG11 and partial structure analysis of the polymer. Appl. Env. Microbiol. 60: 3914-3919.

Willey JM, Van der Donk WA. 2007. Lantibiotics: peptides of diverse structure and function. An. Rev. Microbiol., 61: 477-501.

Wouters EHE, Ayad J, Hugenholtz, Smit G. 2002. Microbes from raw milk for fermented dairy products, Int. Dairy J., 12: $91-109$.

Yaygin H. 1992. Kimiz ve Ozellikleri. Yeni Matbaa : Antalya, Turkey ; p. 69.

Yeaman MR, Yount NY. 2007. Unifying themes in host defence effector polypeptides. Nat. Rev. Microbiol., 5: 727-740. 